This is a postprint version of the following published document:

Katia Bertoldi, Vincenzo Vitelli, Johan Christensen, and Martin van Hecke, 2017. Flexible mechanical metamaterials. Nature Reviews Materials, 2 (17066), pp. 1-11

DOI: https://doi.org/10.1038/natrevmats.2017.66

(C) 2017 Macmillan Publishers Limited, part of Springer Nature. All rights reserved. 


\title{
Flexible mechanicalmetamaterials
}

\author{
Katia Bertoldi, ${ }^{1}$ Vincenzo Vitelli, ${ }^{2,3}$ Johan Christensen, ${ }^{4}$ and Martin van Hecke ${ }^{5,6}$ \\ ${ }^{1}$ Harvard John A. Paulson School of Engineering and Applied Sciences, Harvard University, Cambridge, Massachusetts \\ 02138, USA \\ ${ }^{2}$ Instituut-Lorentz, Universiteit Leiden, Postbus 9506, 2300 RA Leiden, The Netherlands
}

3 James Franck Institute and Department of Physics, The University of Chicago, Chicago, IL 60637, USA

4Instituto Gregorio Millán Barbany, Universidad Carlos III de Madrid, Avenida de la Universidad 30, 28916 Leganés

(Madrid), Spain

5 AMOLF, Science Park 104, 1098 XG Amsterdam, The Netherlands

${ }^{6}$ Huygens-Kamerlingh Onnes Laboratories, Universiteit Leiden, Postbus 9504, 2300 RA Leiden, The Netherlands

Corresponding author: Johan Christensen, johan.christensen@uc3m.es

\begin{abstract}
Mechanical metamaterials exhibit properties and functionalities that cannot be re- alized in conventional materials. Originally the field focused on achieving unusual (zero or negative) values for familiar mechanical parameters, such as such as density, Poisson ratio or compressibility, but more recently new classes of metamaterials - in- cluding shape-morphing, topological and nonlinear metamaterials have emerged. These materials exhibit exotic functionalities, such as pattern and shape transforma- tions in response to mechanical forces, unidirectional guiding of motion and waves, and reprogrammable stiffness or dissipation. In this Review, we identify the design princi- ples leading to these properties, discussing in particular linear and mechanism-based metamaterials (such as origami- and kirigami-based metamaterials), metamaterials har- nessing instabilities and frustration and topological metamaterials. We conclude by outlining the future challenges for the design, creation and conceptualization of advanced mechanical metamaterials.
\end{abstract}

\section{[H1] Introduction}

Metamaterials are carefully structured materials - often consisting of periodically arranged building 
blocks - that exhibit properties and functionalities that differ from and surpass those of their constituent materials, rather than simply combining them. In the past decades, metamaterials that manipulate optical ${ }^{1}$, acoustic $^{2}$ and thermal ${ }^{3}$ fields and that have highly unusual properties, such as a negative index of refraction, have been demonstrated. This has led to new applications, such as perfect lenses ${ }^{4}$.

Mechanical metamaterials constitute a more recent branch of metamaterials research that exploit motion, deformations, stresses and mechanical energy ${ }^{5,6}$ (FIG. 1). Although they borrow design ideas from wavebased metamaterials, they also provide new challenges and opportunities. For example, both wave-based and mechanical metamaterials can use geometry to obtain zero or negative material parameters ${ }^{7-18}$, but in the context of mechanical metamaterials several recent designs also harness shape morphing ${ }^{19-35}$, topological protection $^{36-61}$, and instabilities and nonlinear responses ${ }^{62-74}$ to obtain advanced functionalities. Auxetic materials - materials that either expand or contract in all directions when a force is applied - are an early example of mechanical metamaterials and illustrate well how structure controls the behaviour of mechanical metamaterials

(BOX 1).

The building blocks of mechanical metamaterials, the meta-atoms, deform, rotate, buckle, fold and snap in response to mechanical forces, and are designed such that adjacent building blocks can act together to yield the desired collective behaviour. A cornerstone for the design of both meta-atoms and metamaterials are slender elements (FIG.1). As the bending stiffness of these elements scales with the third power of their thickness, very strong stiffness heterogeneities can be designed and realized by using various additive manufacturing techniques. These heterogeneities underlie the qualitative differences between the global properties of metamaterials and those of their constituent materials, thus carefully designed architectures can be used to achieve any combination of linear elastic coefficients that is not forbidden by thermodynamics. Often such heterogeneities are used to approximate hinging elements, leading to nearlyfreely hinging structures featuring floppy modes and mechanisms ${ }^{75}$. As we discuss in this Review, a wide variety of metamaterial designs exploit this principle, in particular the origami, kirigami and shape-shifting materials. Slender elements allow for large deformations leading to geometric nonlinearities, and are prone to elastic instabilities such a buckling and snapping (FIG. 1). Nonlinearities and instabilities underlie a number of advanced metamaterial functionalities, such as multistability and programmability. Finally, topological phases have recently been created in suitably designed mechanical structures. The properties of these metamaterials are topologically protected, that is, they are robust against smooth deformations of the material. We conclude the Review by outlining the key challenges for future work. 


\section{[H1] Linear mechanical metamaterials}

[H3] Linear elastic metamaterials. Whereas isotropic materials are described by two elastic coefficients, such as the Poisson's ratio and Young's modulus, for anisotropic materials the elasticity tensor can contain up to 21 independent coefficients, providing a large target space for mechanical metamaterials design. This is because the basic quantities in mechanics, stress $\left(\sigma_{\bar{j}}\right)$ and strain $\left(e_{k l}\right)$, are symmetric $3 \times 3$ tensors, related via the rank four elastic tensor $C_{i j k}$ :

$$
\sigma_{i j}=C_{i j k l} e_{k l}
$$

In 1995, Milton and Cherkaev showed that it is possible to design architectures consisting of ordinary elastic materials and vacuum to create metamaterials with any form of $C_{i j k l}$ that is not forbidden by thermodynamics ${ }^{10}$. Auxetic materials are one example of metamaterials with unusual $C_{i j k l}$. Extremal materials, which only resist one type of de- formation (BOX 1), and pentamode materials - a particular type of extremal materials - constitute another example. Pentamode materials are defined by having five of the six eigenvalues of the elasticity tensor in the Mandel notation equal to zero, which implies that most $C_{i j k l}$ coefficients are zero ${ }^{10}$. A striking example of a pentamode material is a structure for which the ratio of bulk-to-shear elastic modulus diverges: just like a fluid, such a material does not resist shear and only resists volumetric deformations ${ }^{10}$. Advances in additive manufacturing have enabled the creation of $3 \mathrm{D}$ extremal materials, ${ }^{11}$ which have been used to produce 'unfeelability cloaks ${ }^{13}$. By layering different extremal materials, metamaterials with any desired $C_{i j k l}$ can be created ${ }^{10}$.

[H3] Heterogeneity. The fundamental reason why architecturing materials leads to new properties is the resulting heterogeneity of stresses and deformations, which causes the breakdown of the affine assumption, which conjectures that deformations are uniform, like in a homogeneous rubber sample. ${ }^{76}$.

For example, for auxetic and extremal metamaterials most deformations are localized at the hinges, such that the global response of the material is entirely different from the local behaviour of its constituents. Such qualitative differences between the constituents and the collective are crucial for a wide variety of heterogeneous, structured media, such as foams ${ }^{77}$, granular media ${ }^{78}$, fibrous materials ${ }^{79}$ and random spring networks ${ }^{80-83}$. For instance, loosely connected networks of linear springs have a much smaller elastic response than that expected from a dimensional argument estimating the elastic modulus from the springs' constant and characteristic length. In particular, the elastic response vanishes at a critical but finite value of the connectivity, for which the network is strongly nonaffine ${ }^{75,80-83}$. This dependence of the bulk elastic properties on the geometry of the network enables the design of geometries that result in specific elastic properties. By mimicking the geometry of jammed particle packings, networks with a vanishing 
shear- to-bulk modulus ratio can be obtained ${ }^{82}$, whereas targeted spring pruning has been used to achieve auxetic random spring networks with a diverging shear-to-bulk modulus ratio ${ }^{84}$. In all cases, subtle changes in the geometry of the disordered networks lead to qualitatively different deformations and elastic properties, which illustrates that the relation between architecture and collective properties is rich and complex.

\section{[H1] Mechanism-based metamaterials}

Mechanisms are collections of rigid elements linked by flexible hinges whose geometric design allows for a zero-energy, free motion. The designability of arbitrarily complex motions by a careful choice of the geometry of the linked elements ${ }^{85}$ makes mechanisms central to a wide range of engineering structures (levers, pulleys, linkages, gears), as well as a popular target for shape-transforming art [https://vimeo.com/36122966]. Mechanisms also underlie a wide variety of mechanical metamaterials (FIG. 2). For example, a collection of squares linked at their tips form an auxetic structure (FIG. 2a), as they can undergo a free hinging motion that causes the structure to uniformly contract or expand. This design has inspired a range of soft metamaterials, as discussed below ${ }^{17,62,66,86-89}$. Plates linked by flexible hinges form origami metamaterials. The so-called Miura-ori structure (FIG 2b), which was originally designed to obtain deployable solar panels for applications in space ${ }^{19}$, constitutes the starting point for a range of more complex shape- shifting and programmable origami metamaterials $14,15,19-25,27,29,32,32,34,68,71,90-92$. Finally, an asymmetric mechanism consisting of linked bars that allows motions propagating from left to right but not from right to left was recently demonstrated, and is a prime example of a topological mechanical metamaterial (FIG. 2c) ${ }^{38}$.

[H3] Origami-based metamaterials. The aesthetically pleasant patterns and shapes enabled by origami (from the Japanese 'ori', fold and 'kami', paper) and kirigami (from 'kiru', cut and 'kami', paper) have long been admired, and origami-inspired geometries have been used to obtain deployable structures ${ }^{19}$, flexible medical stents $^{90}$ and flexible electronic devices ${ }^{91}$. Origami also provides a powerful platform for designing mechanism- based metamaterials, starting from 2D sheets with pre-defined crease patterns, such as the Miura-ori structure and its variants $19,22,23,27,32,68$, the square twist ${ }^{71}$ and the box-pleat tiling ${ }^{20}$ (FIG. 3). These 'metasheets' can serve as auxetic metamaterials 14,22 or be smoothly modified to fold into arbitrary shapes $^{32}$. Because origami-based mechanisms often feature multiple discreet folding motions, with only one continuous degree of freedom, they enable the design of multishape metamaterials 27 . Moreover, exploring the competition between bending and hinging energies 24,71 allows the design of multi- stable and programmable materials $27,32,68,71$. Finally, fully 3D cellular metamaterials can be designed by stacking folded 
layers $^{14}$ and assembling them in tubes ${ }^{15,21,25,29}$ (FIG. 3a), or by taking inspiration from snapology, $34,35,92$ a modular origami technique (FIG. 3b).

[H3] Kirigami. Kirigami-inspired metamaterials are produced by introducing arrays of cuts into thin sheets of a material (FIG. 3c). This allows the control of the elastic properties of the material ${ }^{31}$ and the achievement of extremely large strains and shape changes 16,26 . The kirigami design principle has been exploited totransform one-atom-thick graphene sheets into resilient and movable structures ${ }^{93}$, to engineer elasticity in stiff (almost rigid) nanocomposites without compromising their electrical conductance ${ }^{28}$ and to design stretchable lithium-ion batteries ${ }^{94}$ and diffraction gratings with tunable periodicity ${ }^{95}$. Furthermore, kirigami-patterned sheets exhibit out-of-plane deformations, which provides a route to form complex 3D mesostructures ${ }^{72,96}$ and enables planar solar tracking ${ }^{97}$. Finally, by combining origami and kirigami, the design space can be greatly expanded ${ }^{98}$ and complex surfaces can be realized 99 .

[H3] Soft mechanism-based metamaterials. The geometric design of mechanisms can also be levered to create soft mechanism-based metamaterals that have slender, flexible parts as hinges, connecting stiffer elements that can easily rotate. The low-energy deformations of these metamaterials closely shadow the free motion of the underlying mechanism. External forces easily excite these soft modes, which generally have a specific spatial structure, very different from the smooth deformations of ordinary elastic media. This allows the design of soft metamaterials which undergo programmable shape shifting,

ranging from auxetic materials in $2 D^{62,66,86-89}$ and $3 D^{17,30,100}$ to size-morphing spheres

that can be used as reversible encapsulation systems ${ }^{64}$ (FIG. 4a). Moreover, using combinatorial techniques to connect different unit cells, it was recently shown that aperiodic mechanical metamaterials that exhibit precisely pre-programmable shape changes can be rationally designed ${ }^{30}$ (FIG. $4 \mathrm{~b}$ ). In all these examples, as mentioned, the hinges are slender beams: as discussed in the next section, this can be exploited to obtain nonlinear, collective buckling phenomena under compression.

\section{[H1] Instability-based metamaterials}

Elastic instabilities and large deformations enable the achievement of strongly non- linear relations between macroscopic stresses and strains, even if the material remains in the near-linear regime. Hence, a variety of mechanical metamaterials are made of meta-atoms that give access to these nonlinearities. Slender elements can create large deformations in response to small forces, which leads to so-called geometric nonlinearities. Symmetric slender elements can undergo buckling instabilities that result in strong but reversible nonlinearities under precisely designable loading conditions. Finally, many elastic structures 
feature two stable states connected by a rapid and irreversible 'snap-through' instability (FIG. 1). Creating mechanical metamaterials by assembling these nonlinear and multistable building blocks leads to a range of completely new functionalities.

[H3] Buckling-basedmetamaterials. Highly porous materialsconsisting of networks of beams are ubiquitous in nature and in synthetic structures and devices ${ }^{101}$. Their functionality depends on both the deformation mechanism of the ligaments, which buckle under compression at relatively low strains, and on their microscopic geometry. In disordered elasto-plastic porous materials, buckling of the beam-like ligaments results in irreversible deformations in the form of collapse bands that provide an efficient energy absorbing mechanism ${ }^{102-106}$. In metamaterials comprising regular arrays of elastic beams, buckling may trigger dramatic homogeneous and reversible pattern transformations. The simplest example of such a buckling-based metamaterial is a square array of circular holes embedded in an elastomeric sheet $62,63,107$ (FIG. 5a), which can be seen as an array of rigid domains connected by beams. When the structure is uniaxially compressed, buckling of the beam-like ligaments triggers a sudden transformation of the holes into a periodic pattern of alternating and mutually orthogonal ellipses. Thus, this metamaterial combines the shape-transforming properties of the underlying mechanism of hinged squares with the mechanical functionality of the beam elements that connect these square elements.

For elastic materials, the geometric reorganization triggered by the instability is both reversible and repeatable. Furthermore, it occurs over a narrow range of applied loads, which makes it promising for the creation of materials with properties that can switch in a sudden but controlled manner. This parallel and cooperative buckling leading to a predictable transformation between different microstructures can be instrumental for the design of materials with tunable properties, including systems with tunable negative Poisson's ratio ${ }^{63,108}$ and effective negative swelling ratio ${ }^{18}$, phononic ${ }^{109-111}$ and photonic ${ }^{112}$ switches and colour displays ${ }^{113}$, and systems with switchable chirality ${ }^{114}$. By breaking the symmetry of the underformed pattern, that is, by substituting circular holes with elliptic holes, the sharp buckling transformation is smeared out, leading to a tunable nonlinearity of the effective constitutive law, which has been leveraged to control the buckling behaviour of metabeams (which are beams made of a mechanical metamaterial) ${ }^{66}$. In all these examples, the structures recover their initial shape when unloaded.

[H3] Snapping-based instabilities. Elastic beams may also snap between two different stable configurations, retaining their deformed shape after unloading 67,115,116. Because bistable elastic beams can lock in most of the energy provided to the system during loading, they have been recently used to create fully elastic and reusable energy-trapping metamaterials ${ }^{33,70,117}$ (FIG. 5b). Moreover, the ability of bistable beams of releasing stored elastic energy has been exploited to overcome both dissipative and dispersive effects to 
allow the propagation of mechanical signals with a large amplitude in soft systems made of dissipative materials $^{73}$. Finally, whereas most instability-based meta-materials work only under compressive loadings, a mechanical metamaterial comprising a periodic arrangement of snapping units (consisting of two curved parallel beams that are centrally clamped) undergoes a large extension under tension caused by sequential snap-through instabilities. The material also exhibits a pattern switch from a wavy shape to a diamond configuration $^{74}$.

[H3] Frustrated and programmable metamaterials. An important consideration in the design of metamaterials is whether all building blocks should be able to deform cooperatively, that is, whether the metamaterial should be frustration-free. In general, frustration leads to a complex energy landscape with a plethora of energy minima, which can hinder any desired functionality. However, a controlled amount of frustration can be leveraged to obtain a multistable and programmable behaviour. Perhaps the simplest example are the deformations of flexible origami metamaterials - obtained by 'popping-through' some of the folds - which have been used to obtain a programmable stiffness ${ }^{68}$ (FIG. 6a). Moreover, incompatible folding patterns for which folding necessitates plate bending can be used to obtain a programmable and multistable response 32,71 .

To achieve frustration-free operation in beam networks, all elastic elements should buckle into the most energetically favoured configuration (a half sinusoid), preserving at the same time the angles with their neighbours to minimize the deformation energy. In $2 \mathrm{D}$ this requirement can be easily satisfied on a square lattice, but not on a triangular lattice, so that the system becomes frustrated and favours the formation of complex ordered patterns ${ }^{69}$ (FIG. 6b). More generally, meta-atoms that leverage instabilities and geometric nonlinearities to become bistable or multistable can lead to tunable or programmable metamaterials that adapt their functionality depending on the configuration of their building blocks. For example, an inhomogeneous confinement applied to a biholar metamaterial creates a competition between two micropatterns; this effect has been exploited to realize a complex multistable system with a programmable response $^{65}$ (FIG 6c). Finally, subjecting a shape-morphing metamaterial to incompatible boundary conditions can yield a response that depends nontrivially on the texture of both the material and the boundaries ${ }^{30}$.

\section{[H1]Topological metamaterials}

Topological mechanical metamaterials display properties that are topologically protected. The properties of non-topological metamaterials are sensitive to both random and systematic changes in their microstructure. By contrast, topologically protected properties are not affected by smooth deformations of the underlying geometry or by the presence of disorder. Therefore, topological metamaterials provide an 
exciting path- way towards materials with robust functionalities.

The concept of topological protection is a hallmark of modern condensed matter physics that has a crucial role in quantum Hall systems and electronic topological insulators ${ }^{118}$. Like their counterparts in electronics and photonics, topological mechanical systems can harbour classical states (free motions, load bearing states or vibrations) whose existence is directly linked to the presence of a topological index ${ }^{36-60}$. Topological indexes are typically integer-valued quantities that cannot be changed by continuous deformations of the underlying structure ${ }^{118}$. A simple example is the genus of a surface, a number that counts the number of holes in the surface: from the perspective of topology a doughnut and a tea cup are equivalent, because the two shapes can be smoothly deformed into each other without introducing cuts. In topological metamaterials, the topological indexes do not characterize the structures themselves, but rather their excitations, such as vibrational modes or elastic waves.

[H3] Topological mechanisms and states of self-stress. The simplest topological metamaterials are mechanisms that display a peculiar breaking of inversion symmetry. An example is a structure composed by a chain of rigid rotors connected by rigid beams 37,38 (FIG. 2c). To understand the properties of this linkage (and of many other mechanical structures), it is useful to view them as networks composed of $N_{S}$ sites (such as point masses) connected by $N_{\mathrm{b}}$ central force bonds (such as springs or rigid beams). In $d$ dimensions, the number of degrees of freedom is given by $d N_{S}$ and the total number of constraints is simply $N_{b}$. The number of zero energy modes $n_{m}$, which are soft deformations that at the lowest order cost no energy, is then given by the Maxwell criterion $^{119}$

$$
d N_{\mathrm{S}}-N_{\mathrm{b}}=n_{\mathrm{m}}-n_{\mathrm{sS}}
$$

where $n_{\mathrm{ss}}$ denotes the number of redundant constraints or, equivalently, the number of states of self-stress - these are tensions or compressions applied to the bonds that do not result in net forces on the nodes.

Breaking the left-right symmetry of the unit cell can induce topological polarization, a concept we discuss in more detail later in this section ${ }^{38}$. In simple words, as an electrically polarized material can host localized charges at opposite boundaries, a topologically polarized material can harbour zero modes or states of self-stress at its edges. For example, in a topological chain composed of rigid bars and springs (FIG. 7a), the orientation and length of the bars determines the extension of the springs. Assuming that the springs are neither stretched nor compressed, there is a zero-energy deformation that satisfies the linearized constraint equation $b u_{n+1}-a u_{n}=0$, where $u_{n}$ is the displacement of the $n^{\text {th }}$ node connecting adjacent bars and springs, while $a$ and $b$ are structural parameters characteristic of the geometry of the unit cell 37,38 . Solving this equation yields $u_{n+1}=(a / b) u_{n}$. Thus, depending on whether $a / b$ is bigger or smaller than one, 
displacements are amplified or quenched. Equivalently, a floppy mode (a zero-energy mode) is localized near the right $(a>b)$ of left $(a<b)$ edge of the chain, depending on which of the two directions the bars are leaning in, and the topological polarization points to the right or left, respectively.

In this example, there are no redundant constraints. In the absence of boundaries (for example on a closed ring), this system has as many degrees of freedom (the angles of the rotors) as constraints (the number of beams), and no zero modes. However, for a finite chain (obtained by cutting the ring), one constraint is missing and a zero-energy mode appears in the system: there is no linear restoring force that stops this motion. The Maxwell criterion does not prescribe where this zero mode is located, but a more detailed calculation using the dynamical matrix reveals that the zero-energy eigenmode is localized at the edge towards which the rotors are pointing 37 .

This zero mode requires a system with edges, but its location reflects the breaking of inversion symmetry of the unity cell, which is a bulk property linked to a topological index in the vibrational spectrum ${ }^{37}$. Intriguingly, these localized zero-energy modes can be moved anywhere in the system by exploiting nonlinear domain walls that reconfigure the structure without stretching any of the beams (FIG. 2c) ${ }^{38}$. Smooth deformations of the network leave the left hand side of Eq. 2 unchanged; this means that after such a smooth deformation, the difference between the number of zero modes and states of self-stress remains invariant even if $n_{m}$ and $n_{s s}$ change. The condition $d N_{\mathrm{s}}-N_{\mathrm{b}}=0$ can be viewed as a charge neutrality condition, with the role of positive and negative charges played by zero modes and states of self-stress. The crucial point is that the existence of the zero mode is topologically protected, that is, it persists for a wide range of smooth structural deformations, such as small changes in beam length ${ }^{120}$.

In the following we focus our attention on periodic mechanical structures that are 'charge neutral' but mechanically polarized, so that edge modes can appear at the sample's boundary like charges in an electrically polarized medium. This mechanical polarization is defined in terms of topological invariants of the bulk structure 37 . Just as Gauss's law yields the net electric charge enclosed in a region from the flux of the electric polarization through its boundary, the difference between the number of topological zero modes (the modes that arise without adding or removing bonds anywhere in the structure) minus the number of topological states of self-stress in an arbitrary portion of an isostatic lattice is given by the flux of the topological polarization through its boundary ${ }^{37}$. Thus, domain walls between regions of different polarization can localize zero modes or states of self-stress inside a material as well as at its edges (FIG. 7 shows some examples of macroscopic prototypes). These principles have been used to create stable geared topological metamaterials with protected edge and bulk states (FIG. $7 \mathrm{~b})^{45}$; topological origami that fold more easily from one side (FIG. $7 \mathrm{c})^{48}$; to elucidate how dislocations can localize topological zero modes and 
states of self-stress (FIG. $7 \mathrm{~d})^{40}$; to produce transformable metamaterials that can switch their topological polarization (FIG. 7e $)^{51}$; to generate non-reciprocal mechanical metamaterials analogous to acoustic diodes ${ }^{61}$; and to demonstrate topological control of buckling via states of self-stress in three dimensional cellular metamaterials (FIG. 7f) ${ }^{41}$. The last example shows that states of self-stress are related to the propensity of the structure to locally respond by mechanical failure to stress focusing, in the same way as zero modes can be set in motion if externally activated.

[HB] Topological waves. Besides zero-frequency topological modes, topologically protected modes can also occur at finite frequencies, including those involved in mechanical waves. An example is constituted by the propagation of mechanical waves in the lattices of either coupled gyroscopes ${ }^{42,59}$ or pendula 53 (FIG. $7 \mathrm{~g}$ and $h$ ). The phononic band structure of these systems exhibits gaps - frequency ranges where mechanical waves cannot propagate in the bulk. However, in a finite sample, these gaps are populated by waves that can propagate only at the sample's edge. These phononic edge states are topologically protected in the sense that the waves do not scatter if the shape of the boundary changes (even in the presence of sharp corners, as in FIG. 7h) or if disorder is present ${ }^{121}$.

The phonon localization at the edges is not caused by local variations in material parameters at the boundary. Instead, it is a manifestation of a general and deep physical principle known as the bulk-edge correspondence ${ }^{118}$, which is also at the base of the localization of the zero modes shown in FIG. 7a. The phononic band structure of these metamaterials is characterized by the presence of integer-valued topological invariants, the Chern numbers. The Chern number abruptly changes at the sample's edge, which separates the topological from the normal medium; for the Chern number to change, a gap closing must occur. Thus, as a result of the topological nature of the bulk band structure, gapless edge modes appear, irrespective of the boundary shape.

This mechanism is common to all topological metamaterials (as well as to several classes of electronic and photonic topological insulators). Different physical mechanisms may be responsible for the opening of the gaps in the band structure. For ex- ample, in the gyroscopic lattices 42,59 and in proposed metamaterials based on rotating fluids $43,52,122$, the gap arises from the breaking of time-reversal symmetry. As a result, the wave propagation at the edge is unidirectional and immune to backscattering. The propagation direction of the edge mode is determined by the interplay between the sense of rotation of the microscopic degrees of freedom (the gyroscopes or annuli filled with rotating fluids) and the geometry of the lattice. Particularly exciting is the prospect of self-assembling mechanical topological insulators based on active liquids that flow spontaneously without external drive in confined geometries $^{122}$. The underlying active chiral flow would break time-reversal symmetry, giving rise to robust unidirectional acoustic waveguides at the sample's edges and domain walls. 


\section{[H1] Outlook}

Fuelled by rapid advances in additive manufacturing, computational design and conceptual breakthroughs, research in mechanical metamaterials is bringing about many exciting developments. We close this Review by identifying several challenges for future work.

First, most studies so far have focused on small, pristine samples, but new phenomena will arise in larger samples. Although it is often tacitly assumed that deformations in mechanical metamaterials are essentially homogeneous and follow an idealized de- sign, in practice a finite and perhaps large correlation length is expected, beyond which gradients, domain walls or grain boundaries may arise. Moreover, defects often dominate or alter the material's behaviour, and it is an open question how defects, either resulting from fabrication errors or wilfully introduced 40,68 , change the properties of mechanicalmetamaterials.

Second, although many mechanical metamaterials comprise periodic structures, more advanced, spatially textured functionalities require aperiodic materials. The design of spatially textured metastructures has advanced significantly for origami-based metamaterials; for these structures, computer software such as Treemaker can generate folding patterns that allow the transformation of flat sheets into arbitrary 3D shapes $^{123}$, and perturbative techniques that create folding patterns that approach arbitrary, smooth $3 \mathrm{D}$ surfaces have recently been introduced ${ }^{32}$. For other types of mechanical metamaterials such techniques are generally not available, although a combinatorial strategy for the rational design of aperiodic, yet frustration-

free, shape shifting metamaterials has recently been introduced ${ }^{30}$. Much more work is needed to flesh out these notions into a coherent framework, and many questions remain open. For example, it is not yet clear how to design metamaterials that can morph into a distinct number of predefined shapes.

Third, more complex metamaterials that explore complex energy landscapes and activities are on the horizon. Several examples of frustrated metamaterials that feature multistability and programmability have been recently demonstrated $27,30,65,69$. Rational design of the underlying complex energy landscapes is still in its infancy, but would allow the creation of a host of more complex metamaterials, potentially leading to functionalities such as information storage and retrieval, a prerequisite for more complex programmable materials. If activated by motors or external fields, shapeshifting meta- materials can be used to create useful robotic structures ${ }^{124}$. Deep integration of actuation and amplification of mechanical information is crucial to overcome the inevitable dissipative processes, and if combined with information processing, for example by logic gates ${ }^{73}$, would open the door to truly smart metamaterials.

Fourth, additive manufacturing techniques, from 3D printing to laser cutting and two-photon 
lithography, have had a crucial role in enabling the shaping and patterning of mechanical metamaterials. Many of these techniques are still in their infancy, and the range of base materials that can be printed is still limited. Combining multiple (contrasting) materials is very challenging, but the ability to mix elastic, plastic and viscous materials could lead to completely new classes of mechanical meta-behaviours. Moreover, the inclusion of materials that have specific functionalities may enable hybrid - opto-, thermo- or electro-mechanical for example - classes of metamaterials.

Fifth, the range of scales and aspect ratios that can be currently achieved is limited. Combinations of topdown and bottom-up (self-assembly) techniques may allow the translation of metamaterial concepts to smaller scales, combining graphene origami ${ }^{93}$, colloidal self-assembly ${ }^{125}$ and even chemistry to create designer materials sculpted over a wide range of scales.

Finally, the rational design of metamaterials with a given target property or functionality remains fiendishly difficult, and many designs so far have relied on luck and intuition. For example, Kempe's universality theorem states that it is possible to construct a pantograph-type mechanism that traces out any desired complex motion, but in all but the most simple cases the mathematics produces such complex linkages that the practical applicability of this theorem is limited ${ }^{85}$. We expect that a combination of rational and combinatorial techniques and topological concepts with computer-aided design techniques, such as evolutionary algorithms ${ }^{126}$, will increasingly allow researchers to create mechanical metamaterials with more complex and targeted functionalities.

1 Soukoulis, C. \& Wegener, M. Past achievements and future challenges in the development of threedimensional photonic metamaterials. Nature Photonics 5, 523-530 (2011).

2 Cummer, S. A., Christensen, J. \& Alu, A. Controlling sound with acoustic metamaterials. Nature Reviews Materials 1, 16001 (2016).

3 Schittny, R., Kadic, M., Guenneau, S. \& Wegener, M. Experiments on transformation thermodynamics: Molding the flow of heat. Physical Review Letters 110, 195901 (2013).

4 Pendry, J. B. Negative refraction makes a perfect lens. Physical Review Letters 85, 3966-3969 (2000).

5 Kadic, M., Bückmann, T., Schittny, R. \& Wegener, M. Metamaterials beyond electromagnetism. Reports on Progressin Physics 76, 126501 (2013). 
6 Christensen, J., Kadic, M., Kraft, O. \& Wegener, M. Vibrant times for mechanical metamaterials. $\underline{\text { MRS }}$ Communications 5, 453-462(2015).

7 Lakes, R. Foam structures with a negative poisson's ratio. Science 235, 1038-1040 (1987).

8 Lakes, R. S. Deformation mechanisms of negative poisson's ratio materials: structural aspects. J. Mat. Sci. 26, 2287-2292 (1991).

9 Milton, G. Composite materials with poissons ratio close to -1 . J. Mech. Phys. Solids 40, 1105-1137 (1992).

10 Milton, G. W. \& Cherkaev, A. V. Which elasticity tensors are realizable? Journal of engineering materials and technology 117, 483-493 (1995).

11 Kadic, M., Bückmann, T., Stenger, N., Thiel, M. \& Wegener, M. On the practicability of pentamode mechanical metamaterials. Applied Physics Letters 100,191901 (2012).

12 Nicolaou, Z. G. \& Motter, A. E. Mechanical metamaterials with negative compressibility transitions. Nature Materials 11, 608 (2012).

13 Bückmann, T., Thiel, M., Kadic, M., Schittny, R. \& Wegener, M. An elasto-mechanical unfeelability cloak made of pentamode metamaterials. Nature communications 5, 4130 (2014).

14 Schenk, M. \& Guest, S. D. Geometry of miura-folded metamaterials. Proceedings of the National Academy of Sciences 110, 3276-3281 (2013).

15 Yasuda, H. \& Yang, J. Re-entrant origami-based metamaterials with negative poisson's ratio and bistability. Physical Review Letters (2015).

16 Gatt, R.etal. Hierarchical auxetic mechanical metamaterials. Scientific Reports5, 8395(2015).

17 Babaee, S. et al. 3d soft metamaterials with negative poisson's ratio. Advanced Materials 25, 5044-5049 (2013).

18 Liu, J. et al. Harnessing buckling to design architected materials that exhibit effective negative swelling. Advanced Materials (2016).

19 Miura, K. Method of packaging and deployment of large membranes in space. The Institute of Space and Astronautical Science report 618, 1-9 (1985).

20 Hawkes, E. et al. Programmable matter by folding. Proceedings of the National Academy of Sciences 107, 12441-12445 (2010).

21 Tachi, T. \& Miura, K. Rigid-foldable cylinders and cells. Journal of the International Association for Shell and Spatial Structures (IASS) 226 (2012).

22 Wei, Z. Y., Guo, Z. V., Dudte, L., Liang, H. Y. \& Mahadevan, L. Geometric mechanics of periodic pleated 
origami. Physical Review Letters 110, 215501 (2013).

23 Lv, C., Krishnaraju, D., Konjevod, G., Yu, H. \& Jiang, H. Origami based mechanical metamaterials. Scientific Reports 4 (2014).

24 Lechenault, F., Thiria, B. \& Adda-Bedia, M. Mechanical response of a creased sheet. Physical Review Letters 112, 244301 (2014).

25 Cheung, K. C., Tachi, T., Calisch, S. \& Miura, K. Origami interleaved tube cellular materials. Smart Materials and Structures 23 (2014).

26 Cho, Y. et al. Engineering the shape and structure of materials by fractal cut. Proc. Natl. Acad. Sci. USA 111, 17390 (2014).

27 Waitukaitis, S., Menaut, R., Chen, B. G.-g. \& van Hecke, M. Origami multistability: From singleverticesto metasheets. Physical Review Letters 114,055503(2015).

28 Shyu, T. et al. A kirigami approach to engineering elasticity in nanocomposites through patterned defects. Nature Materials 14, 785 (2015).

29 Filipov, E. T., Tachi, T. \& Paulino, G. H. Origami tubes assembled into stiff, yet reconfigurable structures and metamaterials. Proceedings of the National Academy of Sciences 112, 1232112326 (2015).

30 Coulais, C., Teomy, E., de Reus, K., Shokef, Y. \& van Hecke, M. Combinatorial design of textured mechanical metamaterials. Nature 535, 529-532(2016).

31 Isobe, M. \& Okumura, K. Initial rigid response and softening transition of highly stretchable kirigami sheet materials. Sci. Rep. 6, 24758 (2016).

32 Dudte, L., Vouga, E., Tachi, T. \& Mahadevan, L. Programming curvature using origami tessellations. Nature Materials 15, 583 (2016).

33 Haghpanah, B. et al. Multistable shape-reconfigurable architected materials. Advanced Materials (2016).

34 Overvelde, J. T. B. et al. A three-dimensional actuated origami-inspired transformable meta- material with multiple degrees of freedom. Nature Communications 7 (2016).

35 Overvelde, J., Weaver, J., Hoberman, C. \& Bertoldi, K. Rational design of reconfigurable prismatic architected materials. Nature 541, 347 (2017).

36 Prodan, E. \& Prodan, C. Topological Phonon Modes and Their Role in Dynamic Instability of Microtubules. Physical Review Letters 103, 248101 (2009). URL http://link.aps.org/ doi/10.1103/PhysRevLett.103.248101. 
Kane, C. L. \& Lubensky, T. C. Topological boundary modes in isostatic lattices.

Nature Physics 10, 39-45 (2014). URL http://www.nature.com/doifinder/10.1038/ nphys 2835 .

Chen, B. G., Upadhyaya, N. \& Vitelli, V. Nonlinear conduction via solitons in a topological mechanical insulator. Proceedings of the National Academy of Sciences of the United States of America 111 13004-9 (2014). URL http://www.ncbi.nlm.nih.gov/pubmed/25157161.

39 Vitelli, V., Upadhyaya, N. \& Chen, B. G.-g. Topological mechanisms as classical spinor fields (2014). URL http://arxiv.org/abs/1407.2890v2. 1407.2890.

40 Paulose, J., Chen, B. G.-g. \& Vitelli, V. Topological modes bound to dislocations in mechanical metamaterials. Nature Physics 11, 153-156 (2015). URL http://www.nature.com/doifinder/10.1038/nphys3185.

41 Paulose, J., Meeussen, A. S. \& Vitelli, V. Selective buckling via states of self-stress in topological metamaterials. Proceedings of the National Academy of Sciences 112, 7639-7644 (2015). URL http://www.pnas.org/lookup/doi/10.1073/pnas.1502939112.

42 Nash, L. M. et al. Topological mechanics of gyroscopic metamaterials. Proceedings of the National Academy of Sciences $\quad 112, \quad 14495-14500 \quad$ (2015). URL http://arxiv.org/abs/1504.03362http://www.pnas.org/lookup/doi/10.1073/pnas. 1507413112. 1504.03362.

43 Khanikaev, A. B., Fleury, R., Mousavi, S. H. \& Alù, A. Topologically robust sound propagation in an angular-momentum-biased graphene-like resonator lattice. Nature Communications 6, 8260 (2015). URL http://www.nature.com/doifinder/10.1038/ ncomms9260.

44 Susstrunk, R. \& Huber, S. D. Observation of phononic helical edge states in a mechanical topological insulator. Science 349, 47-50 (2015). URL http://www.sciencemag.org/cgi/ doi/10.1126/science.aab0239.

45 Meeussen, A. S., Paulose, J. \& Vitelli, V. Topological design of geared metamaterials. Physical Review X 6, 041029 (2016).

46 Rocklin, D. Z., Chen, B. G.-g., Falk, M., Vitelli, V. \& Lubensky, T. C. Mechanical Weyl Modes in Topological Maxwell Lattices. $\quad$ Physical Review Letters 116, 135503 (2016). URL http://arxiv.org/abs/1510.04970 http://link.aps.org/doi/10.1103/PhysRevLett.116.135503.1510.04970. 
47 Kariyado, T. \& Hatsugai, Y. Manipulation of Dirac Cones in Mechanical Graphene (2015).

URL http://arxiv.org/abs/1505.06679. 1505.06679.

48 Chen, B. G.-g. et al. Topological Mechanics of Origami and Kirigami. Physical Review Letters

116,135501 (2016). URL http://arxiv.org/abs/1508.00795http://link.aps.org/doi/

10.1103/PhysRevLett.116.135501. 1508.00795.

49 Mousavi, S. H., Khanikaev, A. B. \& Wang, Z. Topologically protected elastic waves in phononic metamaterials. Nature Communications 6, 8682 (2015). URL http://arxiv.org/ abs/1507.03002http://www.nature.com/doifinder/10.1038/ncomms9682. $\quad 1507.03002$.

50 Xiao, M., Chen, W.-J., He, W.-Y. \& Chan, C. T. Synthetic gauge flux and Weyl points in acoustic systems. Nature Physics 11, 920-924 (2015). URL http://www.nature.com/doifinder/ 10.1038/nphys3458.

51 Rocklin, D. Z., Zhou, S., Sun, K. \& Mao, X. Transformable topological mechanical metamaterials 1040 (2015). URL http://arxiv.org/abs/1510.06389. 1510.06389.

52 Yang, Z. et al. Topological Acoustics. Physical Review Letters 114, 1-4 (2015). URL http://ink.aps.org/doi/10.1103/PhysRevLett.114.114301.

53 Süsstrunk, R. \& Huber, S. D. Classification of topological phonons in linear mechanical metamaterials. Proc. Natl. Acad. Sci. 113, E4767-E4775 (2016).

54 Deymier, P. A., Runge, K., Swinteck, N. \& Muralidharan, K. Torsional topology and fermionlike behavior of elastic waves in phononic structures. Comptes Rendus Mécanique 1, 1-12 (2015). URL http://linkinghub.elsevier.com/retrieve/pii/S1631072115000789.

$55 \mathrm{Bi}, \mathrm{R}$. \& Wang, Z. Unidirectional transport in electronic and photonic weyl materials by dirac mass engineering. Phys. Rev. B 92, 241109 (2015). URL https://link.aps.org/doi/ 10.1103/PhysRevB.92.241109.

56 Berg, N., Joel, K., Koolyk, M. \& Prodan, E. Topological phonon modes in filamentary structures. Physical Review E 83, 021913 (2011). URL http://link.aps.org/doi/10.1103/ PhysRevE.83.021913.

57 Peano, V., Brendel, C., Schmidt, M. \& Marquardt, F. Topological Phases of Sound and Light. Physical Review X 5, 031011 (2015). URL http://link.aps.org/doi/10.1103/PhysRevX.5. 031011.

58 Wang, Y.-T., Luan, P.-G. \& Zhang, S. Coriolis force induced topological order for classical mechanical vibrations. New Journal of Physics 17, 073031 (2015). URL http://stacks.iop. org/13672630/17/i=7/a=073031?key=crossref.35acf3822edb030aced42dfe0b6025d2.

59 Wang, P., Lu, L. \& Bertoldi, K. Topological Phononic Crystals with One-Way Elastic Edge Waves. Physical Review Letters 115, 104302 (2015). URL http://link.aps.org/doi/10. 
1103/PhysRevLett.115.104302.

60 Po, H. C., Bahri, Y. \& Vishwanath, A. Phonon analog of topological nodal semimetals.

Phys. Rev. B 93, 205158 (2016). URL https://link.aps.org/doi/10.1103/PhysRevB.93.

205158

61 Coulais, C., Sounas, D. \& Alu, A. Static non-reciprocity in mechanical metamaterials. Nature 542,461 (2017).

62 Mullin, T., Deschanel, S., Bertoldi, K. \& Boyce, M. C. Pattern transformation triggered by deformation. Physical Review Letters 99, 084301 (2007).

63 Bertoldi, K., Reis, P. M., Willshaw, S. \& Mullin, T. Negative poisson's ratio behavior induced by an elastic instability. Adv. Mat. 22, 361-366 (2010).

64 Shim, J., Perdigou, C., Chen, E., Bertoldi, K. \& Reis, P. Buckling- induced encapsulation of structured elastic shells under pressure. Proceedings of the National Academic of Science of the United States of America. $\quad 109$, 5978-5983 (2012).

65 Florijn, B., Coulais, C. \& van Hecke, M. Programmable mechanical metamaterials. Physical Review Letters 113, 175503 (2014).

66 Coulais, C., Overvelde, J. T. B., Lubbers, L. A., Bertoldi, K. \& van Hecke, M. Discontinuous buckling of wide beams and metabeams. Physical Review Letters 115, 044301 (2015).

67 Fargette, A., Neukirch, S. \& Antkowiak, A. Elastocapillary Snapping: Capillarity Induces Snap-Through Instabilities in Small Elastic Beams. Phys. Rev. Lett. 112, 137802 (2014).

68 Silverberg, J. L. et al. Using origami design principles to fold reprogrammable mechanical metamaterials. Science $345,647-650$ (2014).

69 Kang, S. H. et al. Complex ordered patterns in mechanical instability induced geometrically frustrated triangular cellular structures. Physical Review Letters 112, 098701 (2014).

70 Shan, S. et al. Multistable architected materials for trapping elastic strain energy. Advanced Materials 27, 4296 (2015).

71 Silverberg, J. L. et al. Origami structures with a critical transition to bistability arising from hidden degrees of freedom. Nature Materials 14, 389-393 (2015).

72 Zhang, Y. et al. A mechanically driven form of kirigami as a route to $3 \mathrm{~d}$ mesostructures in micro/nanomembranes. Proc. Natl. Acad. Sci. USA 112, 11757 (2015).

73 Raney, J. R. et al. Stable propagation of mechanical signals in soft media using stored elastic 
energy. Proceedings of the National Academy of Sciences (2016).

74 Rafsanjani, A., Akbarzadeh, A. \& Pasini, D. Snapping mechanical metamaterials under tension. Advanced Materials 55, 5931-5935(2007).

75 Alexander, S. Amorphous solids: their structure, lattice dynamics and elasticity. Physics Reports 296, 65 - 236 (1998).

76 van Hecke, M. Jamming of soft particles: geometry, mechanics, scaling and isostaticity. Journal of physics-condesed matter 22, 033101 (2010).

77 Katgert, G., Tighe, B. P. \& van Hecke, M. Jamming of soft particles: geometry, mechanics, scaling and isostaticity. Soft Matter $\mathbf{9}, 9739$ (2013).

78 Jaeger, H. M., Nagel, S. R. \& Behringer, R. P. Granular solids, liquids, and gases. Rev. of Mod. Phys. 68, 1259 (2012).

79 Broedersz, C. P. \& MacKintosh, F. C. Modeling semiflexible polymer networks. Rev. of Mod. Phys. 86, 995 (2014).

80 Jacobs, D. J. \& Thorpe, M. F. Generic rigidity percolation - the pebble game. Phys. Rev. Lett. 75, 4051 (1995).

81 Wyart, M., Liang, H., Kabla, A. \& Mahadevan, L. Elasticity of floppy and stiff random networks. Phys. Rev.Lett.101, 215501(2008).

82 Ellenbroek, W. G., Zeravcic, Z., van Saarloos, W. \& van Hecke, M. Non-affine response: Jammed packings vs. spring networks. EPL 87, 34004 (2009).

83 Ellenbroek, W. G., Hagh, V. F., Kumar, A., Thorpe, M. F. \& van Hecke, M. Rigidity loss in disordered systems: Threescenarios. Phys. Rev.Lett.114,135501(2015).

84 Goodrich, C. P., Liu, A. J. \& Nagel, S. R. The principle of independent bond-level response: Tuning by pruning to exploit disorder for global behavior. Phys. Rev. Lett. 114, 225501 (2015).

85 O'Rourke, J. How to fold it: The mathematics of linkages, origami and polyhedra. Cambridge University Press (2011).

86 Grima, J. N. \& Evans, K. E. Auxetic behavior from rotating squares. Journal of materialsscienceletters 19, 1563(2000).

87 Bertoldi, K., Reis, P. M., Willshaw, S. \& Mullin, T. Negative poisson's ratio behavior induced by an elastic instability. Advanced Materials 22, 361-366 (2010).

88 Overvelde, J. T. B., Shan, S. \& Bertoldi, K. Compaction through buckling in $2 \mathrm{~d}$ periodic, soft and porous structures: Effect of pore shape. Advanced Materials 24, 2337-2342 (2012). 
Overvelde, J. T. B. \& Bertoldi, K. Relating pore shape to the non-linear response of periodic elastomeric structures. Journal of the Mechanics and Physics of Solids 64, 351-366 (2014).

90 Kuribayashi, K. et al. Self-deployable origami stent grafts as a biomedical application of ni-rich tini shape memory alloy foil. Materials Science and Engineering: A 419, 131 - 137 (2006).

91 Song, Z. et al. Origami lithium-ion batteries. Nature Communications 5 (2014).

92 Goldman, F. Using the snapology technique to teach convex polyhedra (CRC Press, 2011).

93 Blees, K. et al. Graphene kirigami. Nature 524, 204 (2015).

94 Song, Z. et al. Kirigami-based stretchable lithium-ion batteries. Scientific Reports 5, 10988 (2015).

$95 \mathrm{Xu}, \mathrm{L}$. et al. Kirigami nanocomposites as wide-angle diffraction gratings. ACS Nano 10, 6156-6162 (2016).

96 Rafsanjani, A. \& Bertoldi, K. Buckling-induced kirigami. Physical Review Letters 118, 084301 (2017).

97 Lamoureux, A. et al. Dynamic kirigami structures for integrated solar tracking. Nat. Comm. 6, 8092 (2015).

98 Eidini, M. \& Paulino, G. H. Unraveling metamaterial properties in zigzag-base folded sheets. Science Advances 1, e1500224 (2015).

99 Sussman, D. et al. Algorithmic lattice kirigami: A route to pluripotent materials. Proc. Natl. Acad. Sci. USA 112, 7449 (2015).

100 Bückmann, T. et al. Tailored 3d mechanical metamaterials made by dip-in direct-laser-writing optical lithography. Advanced Materials 24, 2710-2714 (2012).

101 Gibson, L. \& Ashby, M. Cellular Solids: Structure and Properties (Cambridge University Press, 1999).

102 Wierzbicki, T. \& Abramowicz, W. On the crushing mechanics of thin-walled structures. J. Appl. Mech. 50, 727-734 (1983).

103 Papka, S. \& Kyriakides, S. Biaxial crushing of honeycombs part1 experiments. Int. J. Solids Struct. 36, 4367 - 4396 (1999). URL http://www.sciencedirect.com/science/ article/pii/S0020768398002248.

104 Papka, S. \& Kyriakides, S. In-plane biaxial crushing of honeycombs: Part ii: Analysis. Int. J. Solids Struct. 36, 4397 - 4423 (1999). URL http://www.sciencedirect.com/science/ article/pii/S002076839800225X.

105 Wu, E. \& Jiang, W. Axial crush of metallic honeycombs. Int. J. Impact Eng. 19, 439-456 (1997).

106 Hayes, A. M., Wang, A., Dempsey, B. M. \& McDowell, D. L. Mechanics of linear cellular alloys. Mech. 
Mater. 36, 691-713(2004).

107 Zhang, Y. et al. One-step nanoscale assembly of complex structures via harnessing of an elastic instability. Nano letters 8, 1192-1196 (2008).

108 Babaee, S., Shim, J., Weaver, J., Patel, N. \& Bertoldi, K. 3d soft metamaterials with negative poissons ratio. Advanced Materials 25, 5044-5049 (2013).

109 Bertoldi, K. \& Boyce, M. C. Mechanically-triggered transformations of phononic band gaps in periodic elastomeric structures. Physical Review B77, 052105 (2008).

110 Wang, P., Casadei, F., Shan, S., Weaver, J. \& Bertoldi, K. Harnessing buckling to design tunable locally resonant acoustic metamaterials. Physical Review Letters 113, 014301 (2014).

111 Shan, S. et al. Harnessing multiple folding mechanisms in soft periodic structures for tunable control of elastic waves. Advanced Functional Materials 24, 4935 (2014).

112 Krishnan, D. \& Johnson, H. Optical properties of two dimensional polymer photonic crystals after deformation induced pattern transformations. J. Mech. Phys. Solids 57, 1500-1513 (2009).

$113 \mathrm{Li}$, J. et al. Switching periodic membranes via pattern transformation and shape memory effect. Soft Matter 8, 10322-10328 (2012).

114 Kang, S. et al. Buckling-induced reversible symmetry breaking and amplification of chirality using supported cellular structures. Advanced Materials 25, 3380-3385 (2013).

115 Bazant, Z. P. \& Cedolin, L. Stability of Structures: Elastic, Inelastic, Fracture, and Damage Theories (Oxford University Press, 1991).

116 Pandey, A., Moulton, D., Vella, D. \& Holmes, D. Dynamics of Snapping Beams and Jumping Poppers. EPLEurophys. Lett. 105, 24001 (2014).

117 Restrepo, D., Mankame, N. D. \& Zavattieri, P. D. Phase transforming cellular materials. Extreme Mechanics Letters 4, 52-60 (2015).

118 Hasan, M. Z. \& Kane, C. L. Colloquium : Topological insulators. Rev. Mod. Phys. 82, 3045-3067 (2010). URL http://link.aps.org/doi/10.1103/RevModPhys.82.3045.

119 Lubensky, T. C., Kane, C. L., Mao, X., Souslov, A. \& Sun, K. Phonons and elasticity in critically coordinated lattices. Reports on Progress in Physics 78, 073901 (2015). URL http: //stacks.iop.org/0034-4885/78/i=7/a=073901.

120 Zhou, Y., Chen, B. G.-g., Upadhyaya, N. \& Vitelli, V. Kink-antikink asymmetry and impurity interactions in topological mechanical chains. Phys. Rev. E95, 022202 (2017). URL https:

//link.aps.org/doi/10.1103/PhysRevE.95.022202. 
Mitchell, N. P., Nash, L. M., Hexner, D., Turner, A. \& Irvine, W. T. M. Amorphous gyroscopic topological metamaterials (2016). Arxiv: 1612.09267.

Souslov, A., van Zuiden, B. C., Bartolo, D. \& Vitelli, V. Topological sound in active-liquid metamaterials. Nat.Phys. (2017). URL https://www.nature.com/nphys/journal/vaop/ ncurrent/full/nphys4193.html.

123 Lang, R. J. A computational algorithm for origami design.

Proceedings of Twelfth Annual Symposium on Computational Geometry Location: Philadelphia, PA, U 98 (1996).

124 Felton, S., Tolley, M., Demaine, E., Rus, D. \& Wood, R. A method for building self-folding machines. Science 345, 644-646 (2014). URL http://science.sciencemag.org/content/ 345/6197/644. http://science.sciencemag.org/content/345/6197/644.full.pdf.

125 Zeravcic, Z., Manoharan, V. N. \& Brenner, M. P. Size limits of self-assembled colloidal structures made using specific interactions. $\quad$ Proceedings of the National Academy of Sciences 111, 15918-15923 (2014). URL http://www.pnas.org/content/111/45/15918.abstract. http://www.pnas.org/content/111/45/15918.full.pdf.

126 Miskin, M. Z. \& Jaeger, H. M. Adapting granular materials through artificial evolution. Nature Materials 12, 326 (2013).

127 Gibson, L. J. \& Ashby, M. F. Cellular Solids: Structure and Properties (Cambridge University Press, Cambridge, UK, 1988).

\section{ACKNOWLEDGEMENT}

J.C. acknowledges the support from the European Research Council (ERC) through the Starting Grant 714577 PHONOMETA and from the MINECO through a Ramon y Cajal grant (Grant No. RYC-2015-17156). $\mathrm{MvH}$ acknowledges funding from the Nether- lands Organisation forScientific Research through grant VICI No. NWO-680-47-609.

\section{Author contributions}

All authors contributed equally to the preparation of this manuscript.

\section{Competing interests statement}

The authors declare no competing interests.

Publisher's note

Springer Nature remains neutral with regard to jurisdictional claims in published maps and institutional affiliations.

\section{How to cite this article}


Surname, A. et al. Title. Nat. Rev. Mater. 2, XXXXX (2017). [CE: Please complete]

\section{Further Information box}

Shape-transforming art, https://vimeo.com/36122966.

\section{Box 1 | Auxetic and extremal metamaterials}

Auxetic metamaterials. The application of a uniaxial stretch usually results in a lateral contraction (a stretched rubber band becomes thinner in the transversal direction), thus in this case the ratio between transverse and axial strain, the Poisson's ratio $v$, is positive. For incompressible bulk materials such as rubber, $v$ is roughly $1 / 2$, but if a rubber sheet is patterned with a random arrangement of holes, the effective value of $v$ approaches zero - even though individual rubber filaments get thinner, the overall rubber/vacuum composite assembly does not. This is how random cellular solids, such as cork, achieve nearzero Poisson's ratios, which makes cork a practical material to seal wine bottles, as the upper part of the cork do not expand when the lower part is inserted into the bottle and compressed. It is also possible to design

patterns, such as the inverted hexagon pattern $7,101,127$, that result in auxetic metamaterials, that is, materials with a negative Poisson's ratio, even though the constituent material, rubber, has a positive Poisson's ratio. These examples show that appropriately designed architectures can push metamaterials' properties beyond those of their constituents. In the upper panel of the figure a rubber sheet, a random cellular material and an auxetic metamaterial are shown, before (blue) and after (red) the application of a longitudinal stretch.

Extremal metamaterials. Metamaterials that are designed to resist one particular mode of deformation are called extremal metamaterials ${ }^{10}$. Pin-jointed and inverted honeycomb lattices are examples of extremal materials in $2 \mathrm{D}^{10}$. The bottom panel of the figure shows the theoretical unit cell and the experimental realization of a 3D extremal metamaterial that resists isotropic compression but is very soft against shear in the limit of vanishingly small tips, which approach the limit of ideal hinges, the ratio of shear-to-bulk modulus approaches zero, and the response of the material becomes sim-ilar to that of a fluid.

Lower part of the figure adapted from ${ }^{11}$

FIG. 1: Building blocks of mechanical metamaterials. a| Slender elements, sharp tips and creases localize bending; the bending moment increases monotonically with the angle. b| Elastic beams undergo a buckling instability when axially compressed and fully recover their initial shape when unloaded: this instability provides nonlinear but reversible building blocks for metamaterials. c| Constrained beams can jump to a different equilibrium state through a rapid snap-through buckling. Snapping is often accompanied by bistability, depending on the geometry, amount of confinement and boundary conditions: for example, experiments in which the external deformations are controlled may result in a different response as compared 
with experiments in which the external forces are fixed (dashed and full arrows). Hence, such elements provide bistable building blocks with a hysteretic behaviour.

FIG. 2: Mechanisms-based metamaterials. al A collection of squares linked at their tips can undergo a volume-changing shape transformation, thus it constitutes an auxetic mechanism ${ }^{86}$. b| Rigid plates linked by flexible hinges are at the basis of origami metamaterials. The Miura-ori pattern shown here is a rigidly foldable origami mechanism with one degree of freedom ${ }^{19,22}$. cl Flexibly linked rigid bars form a topologically polarized mechanism. A small motion of the first bar on the right remains localized near the right edge, whereas a larger motion initiates a domain wall that propagates to the left. By contrast, the leftmost bar cannot be moved, thus propagation to the right is not possible ${ }^{38}$.

FIG. 3: Origami-inspired metamaterials. al Deployment and retraction of a 'zipper'-coupled tube system ${ }^{29}$. This origami has only one flexible motion through which it can deform and is very stiff against other deformations. The structure is light weight and can be deployed by acting only on its right end. $b$ | Inspired by snapology, a type of modular origami, a highly flexible mechanical metamaterial with a cubic microstructure was designed; this metamaterial allows for a number of well-defined shape changes and can be folded flat ${ }^{34} \mathrm{cl}$ Combining origami and kirigami, that is, using both cuts and folds, allows to easily shape arbitrary 3D objects starting from 2D sheets ${ }^{99}$. Panel a is adapted from REF. ${ }^{29}$, panel b from REF. ${ }^{34}$, panel c from REF. 99

FIG. 4: Mechanism-based, shape-morphing metamaterials. al The Hoberman Twist-O (top) is a commercial toy that comprises a rigid network of struts connected by rotating hinges. It can easily collapse into a ball measuring a fraction of its original size. This structure is a mechanism, because it has a single continuous degree of freedom. The buckliball (bottom), a structure created by researchers at MIT, is inspired by this popular toy, but applies the mechanism design to an elastic spherical shell. Under pneumatic actuation the buckliball undergoes buckling-induced folding, opening avenues for a new class of active and reversible encapsulation systems ${ }^{64}$. b | A cubic unit cell with a single soft mode can deform into two different shapes that fit together in several ways (right). A combinatorial approach enables the design of a metacube consisting of $10 \times 10 \times 10$ unit cells, each oriented differently in such a way that under uniaxial compression a patterned texture appears on one of the faces of the metacube (left; surface pedestrals are added to aid the visualization of the texture) ${ }^{30}$. Panel a is adapted from REF. ${ }^{64}$, panel b from REF. ${ }^{30}$.

FIG. 5: Instability-based metamaterials. a| A rubber slab patterned with circular holes under-goes a reversible pattern transformation when compressed, as a result of a collective buckling-like instability $62,63,107$. b| Reconfigurable metamaterial containing complex hinge units (inset) that provide multiple kinematic degrees of freedom and multistability ${ }^{33}$. Panel a is adapted from REF. ${ }^{88}$, panel $b$ from REF. 33

FIG. 6: Frustration and tunable metamaterials. a| Each unit cell of the Miura-ori tessellation is mechanically 
bistable. By switching between states, that is, by locally 'popping through' the fold pattern, the compressive modulus of the overall structure can be rationally and reversibly tuned. By virtue of their interactions, these mechanically stable lattice defects also lead to emergent crystallographic structures such as vacancies, dislocations (left) and grain boundaries (right) ${ }^{68}$. b| Similar to how spins can order or assume a frustrated configuration depending on the symmetry of the underlying lattice, buckled beams on frames tend to preserve the angles at joints to minimize the deformation energy. This can be realized for square frames, but not for triangular frames, leading to frustration. In geometrically frustrated cellular structures, buckling triggered under equibiaxial compression results in the formation of complex ordered patterns ${ }^{69} . \mathrm{cl}$ In a metamaterial with alternating large and small holes, a competition between two differently buckled patterns arises if a horizontal confinement is introduced (blue bars) As shown on the plot, the vertical force $F$ as function of the vertical compression $u_{y}$ depends on the degree of confinement. Whereas for small confinement the mechanical response is monotonic, for increasingly strong confinement the response becomes non-monotonic and eventually displays hysteresis: confinement can thus be used to program the mechanical response of the metamaterial ${ }^{65}$. Panel a adapted from REF. ${ }^{68}$, panel b from REF. ${ }^{69}$, panel c from REF. 65 .

FIG. 7: Prototypes of topological mechanical metamaterials. a| Topological chain composed of rigid bars and springs. Depending on the parameters of the system, a floppy mode is localized either at the right- or at the left-hand side of the chain. The topological polarization $\boldsymbol{P}$ is indicated, as well as the displacements $u_{\mathrm{n}}$ and $u_{n+1}$ (red arrows). b| Gears mounted on solid links, connected through joints and arranged into a lattice to form a geared topological metamaterial ${ }^{45}$. $\mathrm{cl}$ Topological origami ${ }^{48}$. dl Zero mode localized at a dislocation on the left of the topological metamaterial and corresponding state of self-stress localized at a dislocation on the right ${ }^{40}$. el Detail of a metamaterial that can switch its topological polarization ${ }^{51}$. $\mathrm{fl}$ Topological state of self-stress localized at a domain wall; under compression, stresses concentrate here, leading to selective buckling ${ }^{41}$. $\mathrm{g}$ | Gyroscopic metamaterial that supports topologically protected chiral edge states false colours represent the phase of a snapshot of a chiral edge wave ${ }^{42} . \mathrm{h} \mid$ A system of suitably coupled pendula (left) provides a mechanical analogue of the quantum spin Hall effect and hosts topologically-protected acoustic waveguides at its edge (right); the blue and red circles represent the polarization of the chiral edge waves $^{44}$. Panel $b$ is adapted from REF. ${ }^{45}$, panel c from REF. ${ }^{48}$, panel d from REF. ${ }^{40}$, panel f from REF. ${ }^{41}$, panel g from REF. ${ }^{42}$, panel $h$ from REF. ${ }^{44}$.

\section{ONLINE ONLY}

Figure Permissions

Figure permissions listed in captions; credit lines for Powerpoint slides is needed.

\section{Subject categories}

Physical sciences / Materials science / Nanoscale materials / Metamaterials

[URI /639/301/357/1015]

Physical sciences / Materials science / Structural materials / Mechanical properties

[URI /639/301/1023/303] 


\section{Web summary}

Mechanical metamaterials exhibit exotic properties that cannot be realized in conventional materials. In this Review, recently emerged shape-morphing, topological and nonlinear metamaterials are discussed, together with their functionalities and design principles, and the future challenges for the field are outlined. 


\section{Author notes}

Please check these figures carefully and return any comments/amendments that you might have to me as soon as possible. In particular, we would like you to check the following:

- Do the figures convey the intended message?

- Are all the labels accurate and in the right place?

- Are all the arrows in the right place?

- Are any chemical structures correct?

- Have shapes and colours been used consistently and accurately throughout the figures?

- Have any of the figures been previously published, or have they been supplied by a colleague(s) who is not a named author on the article?

To mark up any corrections, please use the commenting tools in the PDF, or print and draw by hand, rather than directly editing the PDFs.

Fig. 1

a Slender elements, sharp tips and creases

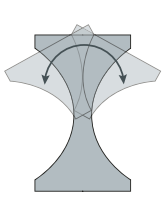

b Elastic beams

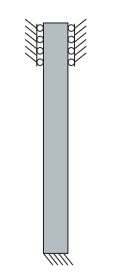

Initial
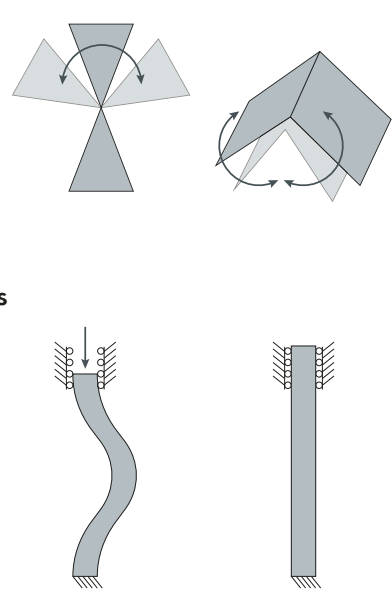

Deformed

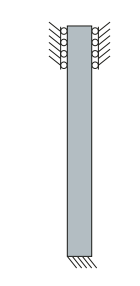

Released
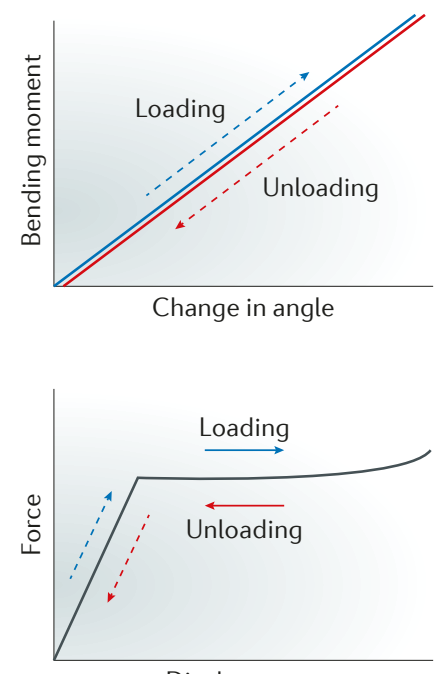

Displacement

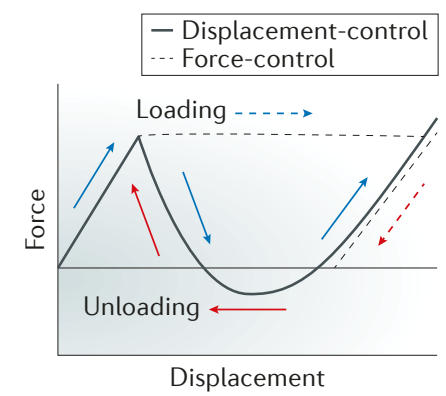

Displacement

C Constrained beams
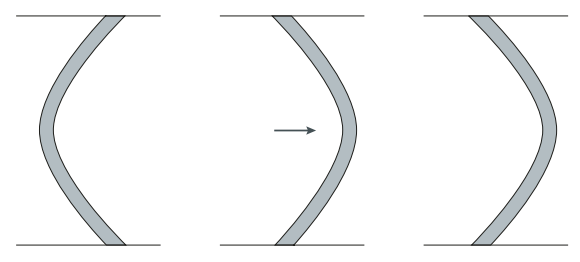
Fig. 2

a Auxetic metamaterial
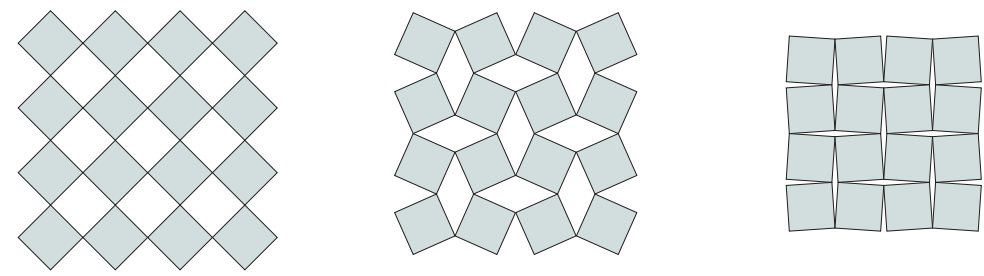

b Origami-inspired metamaterial
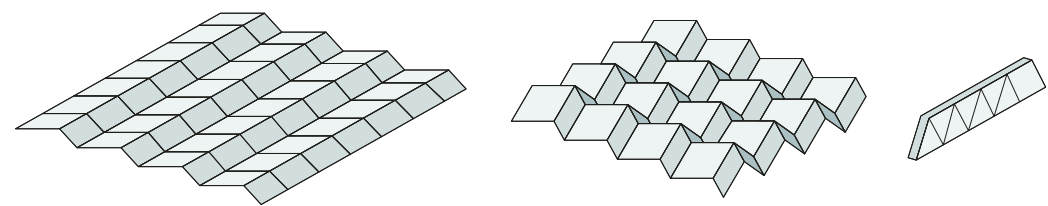

c Topological metamaterial
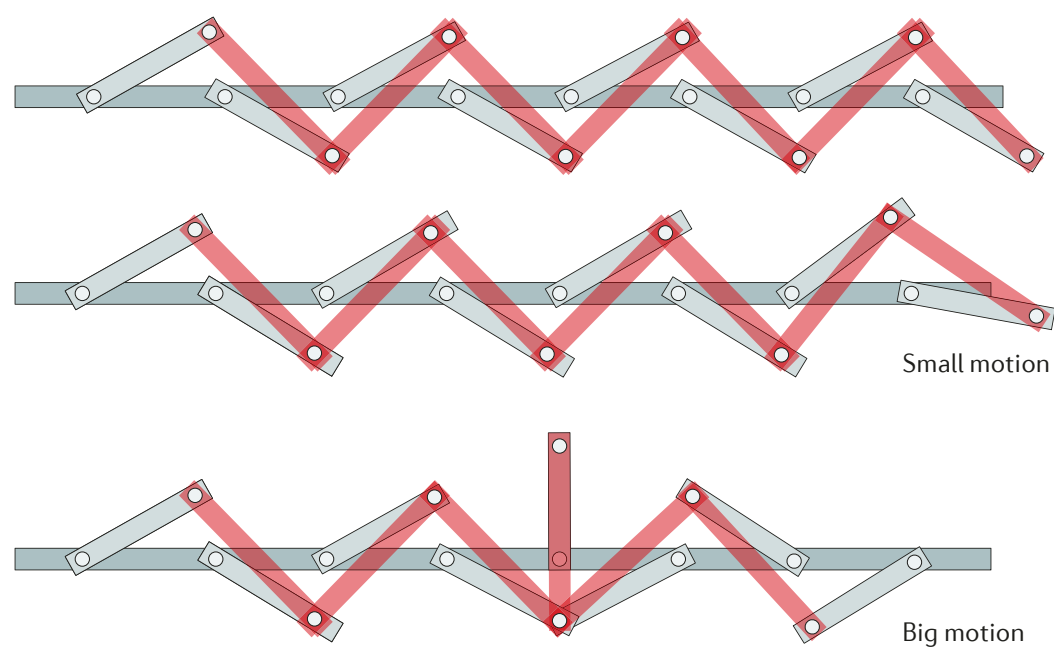
Fig. 3
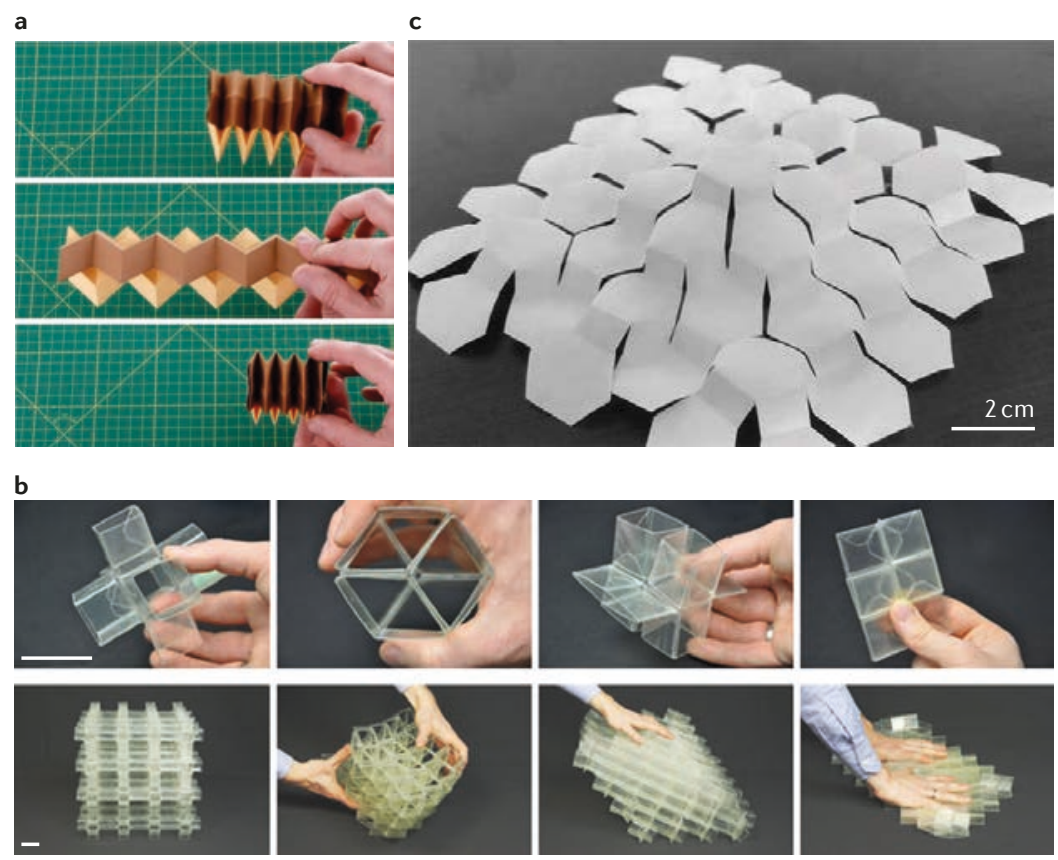

Fig. 4

a Buckling-induced folding
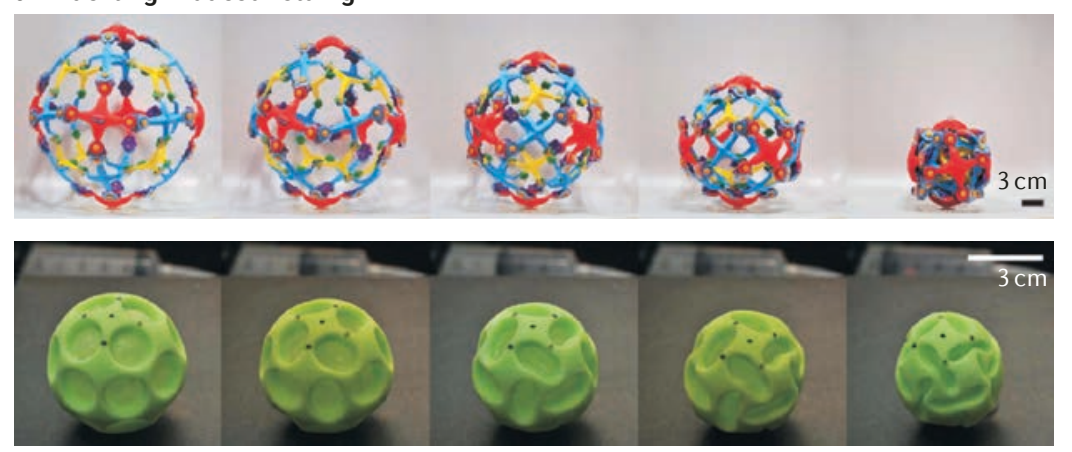

b Metacube obtained with a combinatorial approach
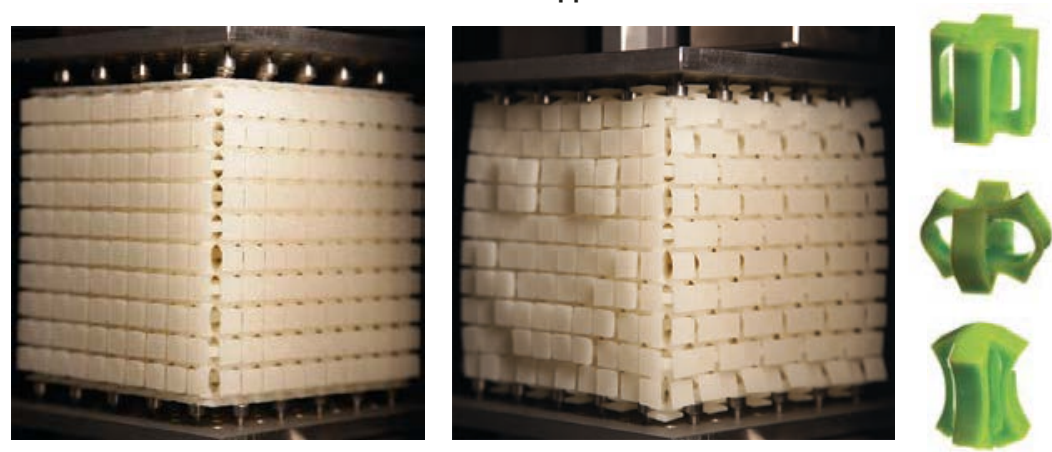
Fig. 5

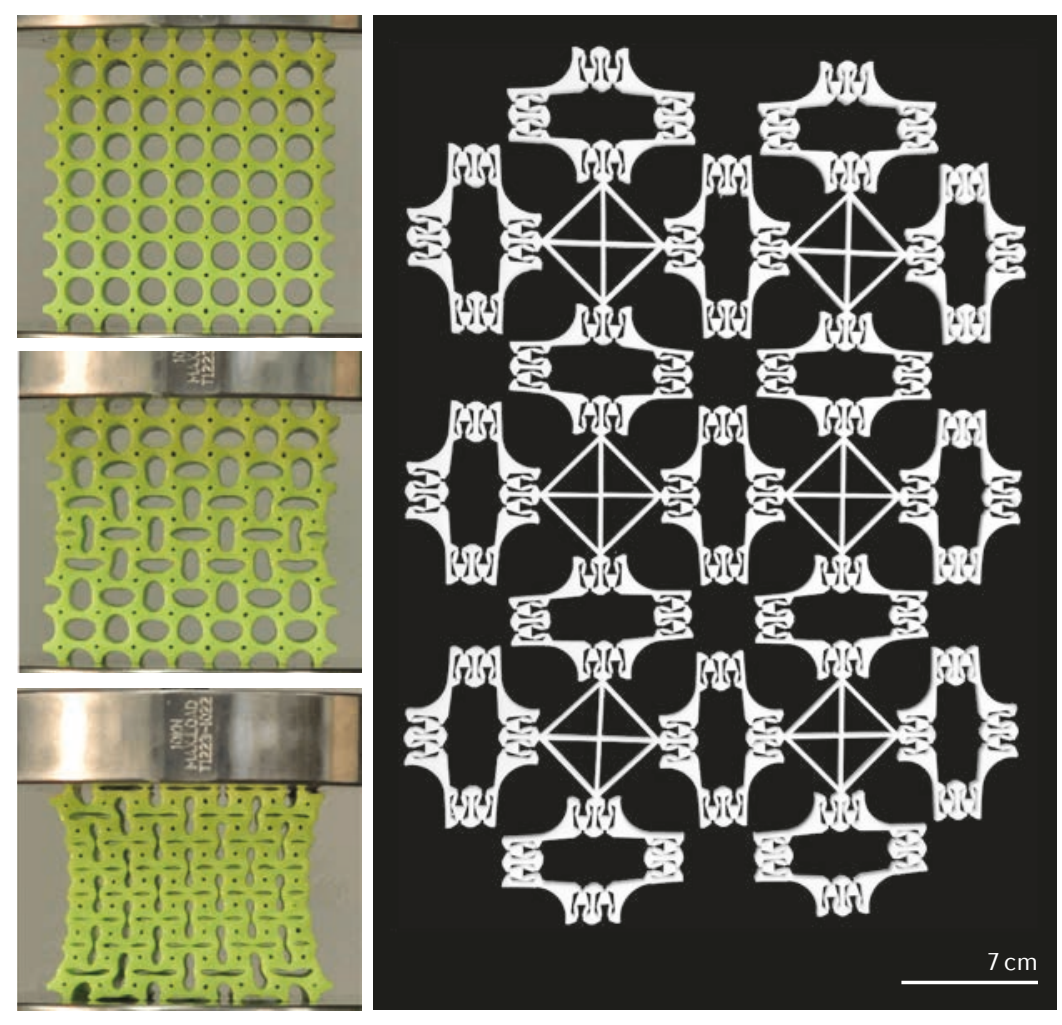

Fig. 6

a Lattice defects
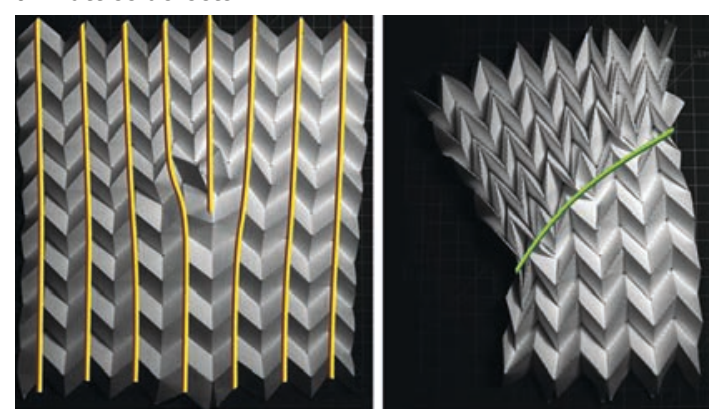

b Geometrically-frustrated structures

Spins
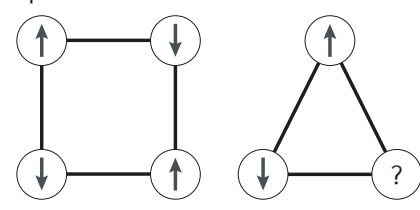

Buckled beams

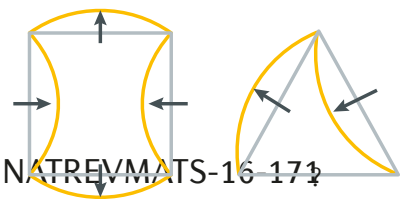

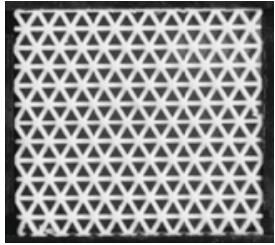

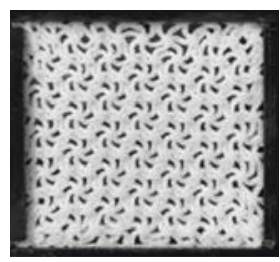

c Dependence on confinement
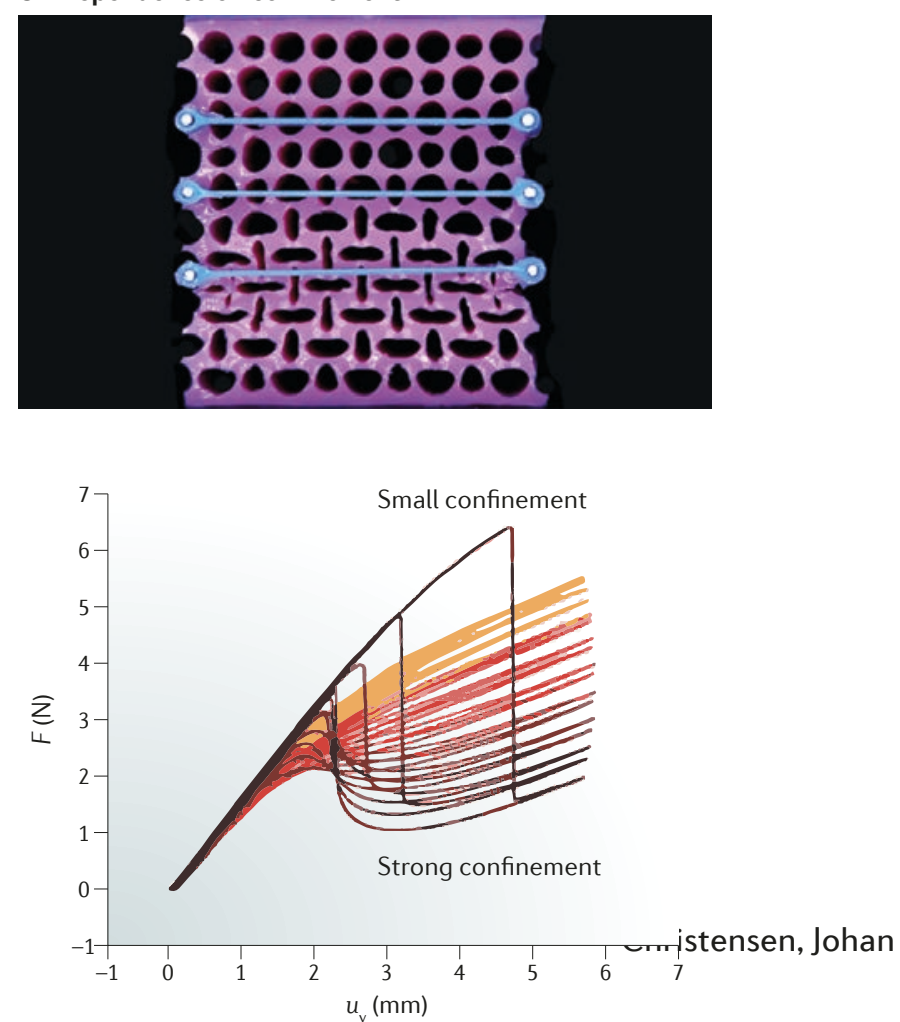

08/09/2017 
Fig. 7

a

$a<b$

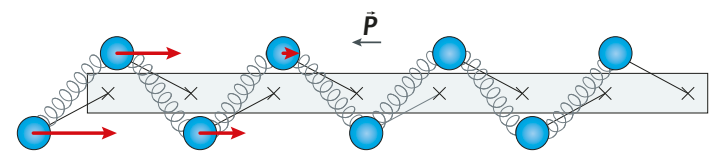

$a>b$

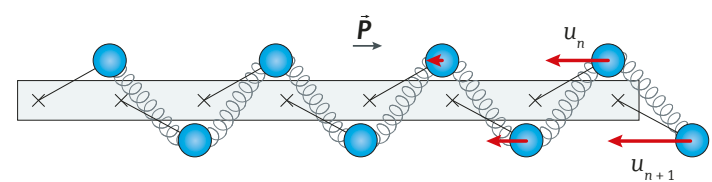

b

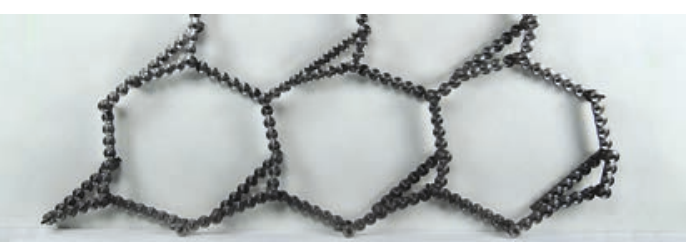

c

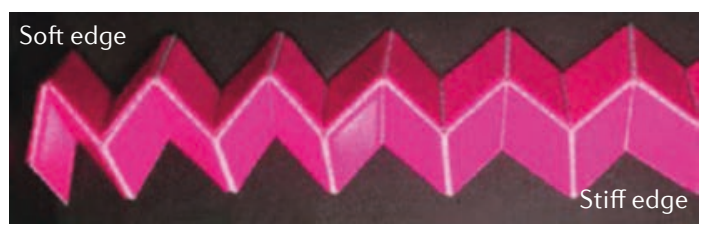

d

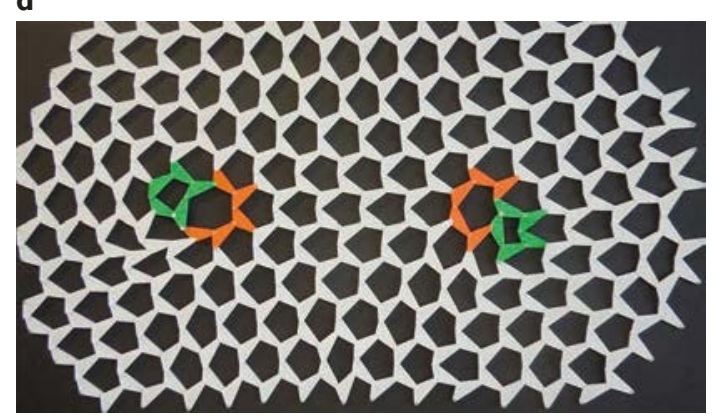

e
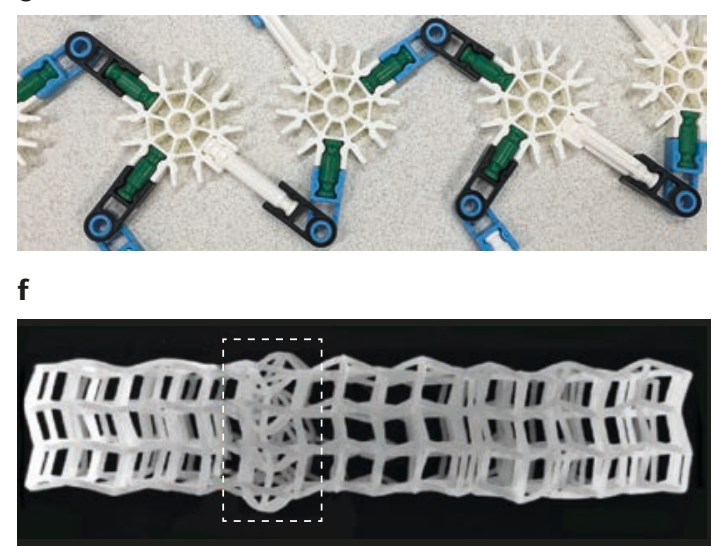

g

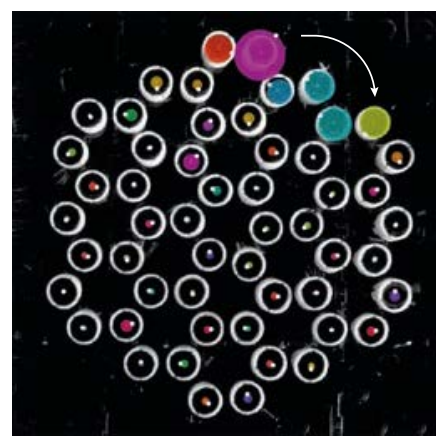

h

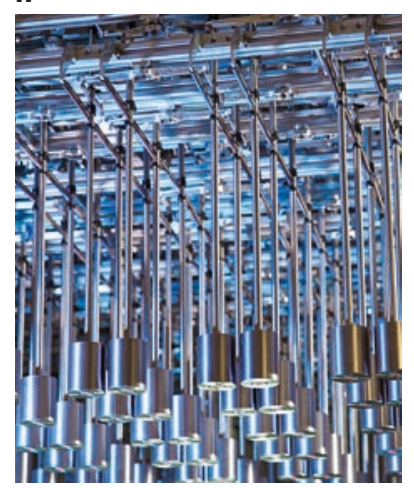

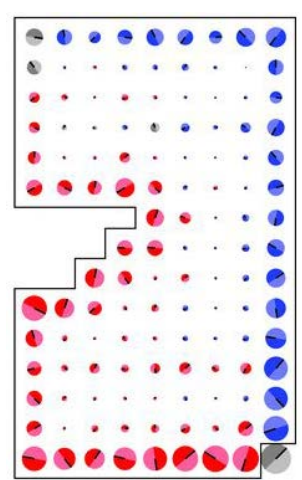


Box 1

a

Positive Poisson's ratio

Zero Poisson's ratio

Negative Poisson's ratio
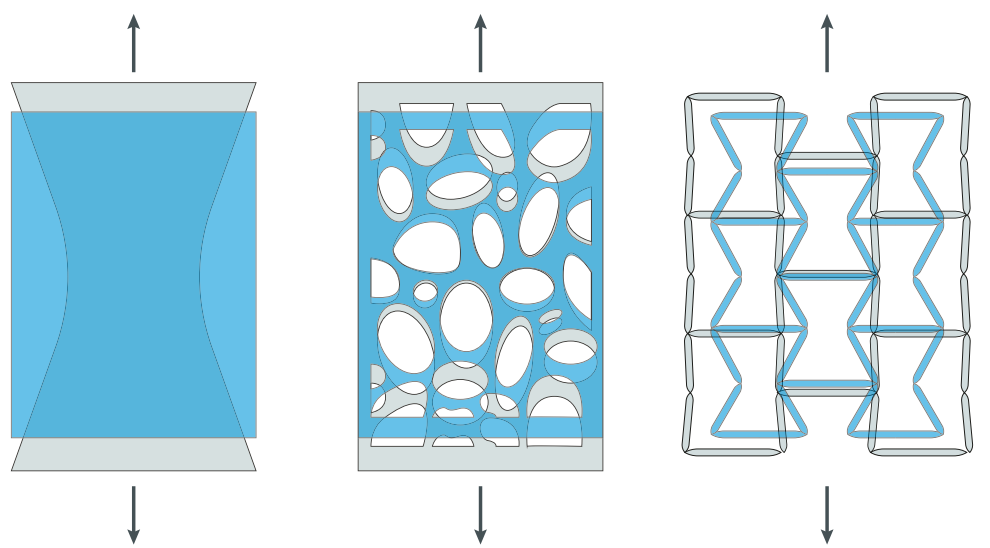

b
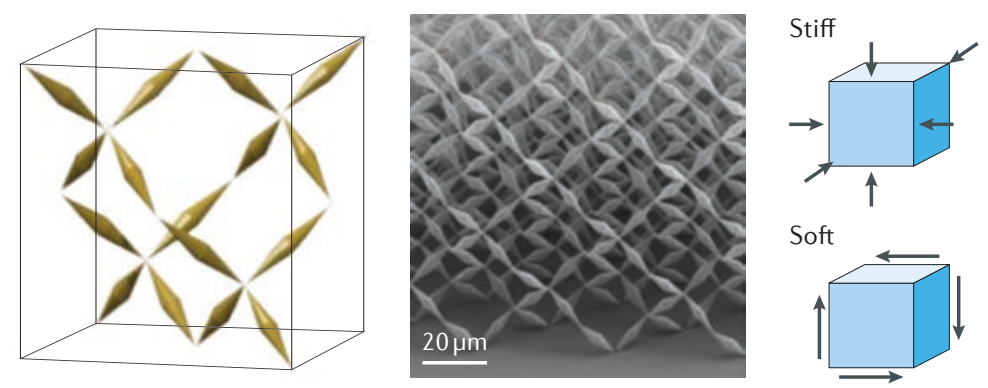